\title{
The Study of Comparing Safety Culture Manual with the Safety Website in Different Presentation Conditions Based on Eye Tracking
}

\author{
Hong-Xia LI \\ College of Management, Xi 'an University of Science \\ and Technology \\ Xi 'an, Shanxi, China \\ e-mail: 406144519@qq.com
}

\author{
Jia-He REN \\ College of Management, Xi 'an University of Science \\ and Technology \\ Xi 'an, Shanxi, China \\ e-mail: 2467606578@qq.com
}

\begin{abstract}
The present study examined the memory effects of different formats and different locations on the safety culture manual and the safety culture website in eye tracking experiments. The results showed: (1) in the memory task, the contents of the manual layout memory effect is better than the web page layout: the content of the paper carrier memory effect is significantly better than the electronic carrier. (2) In the experiment of eye tracking, the number of fixation and the length of fixation in the top part were significantly better than the down part. Memory performance also showed significant difference, the middle and upper part of the memory effect is good.
\end{abstract}

Keywords-safety culture manual; web page content; eye tracking experiments

\section{INTRODUCTION}

China is a major nation of production safety accident in the world. Reasons on production safety accidents happening are complicated, people's lacking of safety awareness, self-help knowledge is not enough, but most importantly the propaganda of safety knowledge is not in place. The spread of safety knowledge is mainly through the safety culture manual and safety culture website. With the rapid development of the Internet, the safety knowledge is more likely to be promoted through the safety culture website. However, which way can play a better propaganda effect?

Compared with research of the traditional paper reading and online reading, some researchers found: when reading paper material, subjects can read faster and have a higher proofreading accuracy and a stronger understanding [1]. Noyes and Garland study shows, the reading of the two carriers involves different modes of memory processing [2]. But there are some studies that different presentation carriers will not affect the reading speed, comprehension and recall [3]. However, Noyes and Garland later discovered that with the improvement of display screen and printing technology, the difference between the two ways of presentation towards to reduce [4]. However, there are a number of studies that the memory effect of advertisement in newspaper format is significantly better than that of network format, the memory effect of paper carrier advertising is better than that of the electronic carrier.

On the content of text, the different positions of the text content have different position effect. A study of the effect of advertising position presentation on the effectiveness of advertising found: (1) People pay more attention to the text at the top of the page, conversely, pay less attention to the text on the bottom of the page; (2) The number of fixations and fixation time of people watching text or patterns on the left are more than that of 
on the right; (3) People pay more attention to the text or pattern at the upper part of the layout than the lower part of the page [5]. Cheng li made an eye movement study on web advertising in different presentation ways and different positions, it turned out that the number of fixations and fixation time on the top and middle of the web pages were significantly more than that in the bottom of the page [6].

According to previous research, the research was just around web advertising or print advertising, website layout. At present, however, the research about layout design of safety culture manual is lacking, and there is no study on the comparison of the propaganda effect of safety culture manual and safety culture website. This study starts with the two main characteristics of the publicity style: format and location, the eye movement experiment was used to compare and analyze the safety culture manual and web pages, to examine influence of different formats and different positions on the propaganda of safety knowledge, to provide an empirical basis for the establishment of a more effective way to promote safety knowledge.

\section{METHOD}

\section{A. Participants}

Those samples chosen for this test are 25 postgraduates, whose uncorrected visual acuity or corrected visual acuity was above 1 , none of them has color blindness and color weakness.

\section{B. Experimental Instrument}

In this experiment, Tobii T60XL eye tracker was used, with an accuracy of 0.5 degrees, a display size of 24 inches, a resolution of $1920 \times 1200$ pixels, a DELL host. In this paper, the experimental procedure use the system software Tobii Studio to present the experiment material, meanwhile, the experimental data are also recorded.

\section{Experimental Materials}

This paper use the pictures of safety culture manual and web page as experimental materials, each format has several pages which come from a coal mine enterprise.

\section{Experimental Design}

This experiment was designed with $2 \times 3$ two-factor. (1) Format factor includes 2 levels: web pages and manuals; (2) Position factor includes 3 levels: top, middle, bottom. The dependent variables were the scores of the subjects and the eye movement data, including the location of fixation point, the length of fixation and the number of fixation.

\section{E. Test Procedure}

First of all, the subjects were asked to sit in front of the eye tracker, and the experimenter explain the experimental requirements to the subjects. Then, the subjects were adjusted to perform eye movement calibration. Finally, we could start the formal experiment.

The experiment includes two parts: reading and answering. First of all, read the instruction. The subjects were asked to browse the experimental materials according to their usual reading habits, and informed that there was a test of reading content after reading. Then start the formal experiment to show the reading material (manual or website content).

After browsing, the subjects were asked to complete two tasks. Task 1: complete recognition test to determine whether or not to see a certain content, if you have seen it, it is judged as "Yes", if not, then "No". Task two: judge the certainty degree of the result. The degree of certainty of the recognition results was evaluated by the four point scale, "Must have seen" scored 4 points, "seems to have seen" scored 3 points, "did not seem to have seen" scored 2 points, "certainly not seen" scored1 points.

\section{F. Processing of Experimental Data}

All the data obtained from the experiment were analyzed by Tobii Studio software. Prior to the analysis, determine the area of interest for each material. The area of interest is the area where the researchers are interested in presented visual stimulation. The experimental data were statistically analyzed with SPSS19.0. 


\section{RESULTS AND ANALYSIS}

\section{A. Analysis on the Memory Effect of Promotional}

\section{Materials in Different Formats}

The results of descriptive statistics (see Table 1) and variance analysis on the memory scores of promotional materials in different formats: the main effect of format factors is significant, $F=11.301, p<0.05$, that is, the memory effect of the manual is better than the memory effect of the web page.

TABLE 1 . THE MEMORY RESULTS OF PROMOTIONAL CONTENT IN DIFFERENT FORMATS

\begin{tabular}{|c|cc|cc|}
\hline Format type & \multicolumn{2}{|c|}{ Manual } & \multicolumn{2}{c|}{ Web page } \\
\hline & $\mathrm{M}$ & $\mathrm{SD}$ & $\mathrm{M}$ & $\mathrm{SD}$ \\
\hline Memory results & 0.85 & 0.33 & 0.52 & 0.29 \\
\hline
\end{tabular}

B. Analysis on the Recognition Results of the

\section{Propaganda Content in Different Positions}

The scores of the recognition results of the propaganda content in different positions are shown in Table 2. The greater the value is, the better the recognition score is.

TABLE 2. RECOGNITION RESULTS OF PUBLICITY MATERIALS AT DIFFERENT POSITIONS

\begin{tabular}{|c|c|c|c|c|}
\hline & & \multicolumn{3}{|c|}{ Position } \\
\hline \multirow{3}{*}{ Manual } & & top & middle & bottom \\
\cline { 2 - 5 } & $\mathrm{M}$ & 2.79 & 3.54 & 1.63 \\
\hline \multirow{2}{*}{ Web page } & $\mathrm{SD}$ & 1.18 & 0.72 & 1.02 \\
\cline { 2 - 5 } & $\mathrm{M}$ & 2.54 & 2.75 & 1.15 \\
\cline { 2 - 5 } & $\mathrm{SD}$ & 1.24 & 1.16 & 0.87 \\
\hline
\end{tabular}

It was found that the main effect of the positions of propaganda content was significantly different through single factor analysis of variance, $\mathrm{F}=5.846, \mathrm{p}<0.01$. Further test found that the recognition scores of the propaganda content in the upper and middle parts were significantly higher than that in the lower position.

\section{An Analysis of Eye Movement Indexes of Different \\ Formats and Locations}

The comparison between the number of fixation and the fixation time of the subjects were shown in table 3.

TABLE 3. THE NUMBER OF FIXATION AND FIXATION TIME OF BROWSING THE PROPAGANDA CONTENT

\begin{tabular}{|c|c|c|c|c|c|}
\hline \multirow{4}{*}{ Format } & \multirow{2}{*}{ Position } & \multicolumn{2}{|c|}{$\begin{array}{c}\text { The number of } \\
\text { fixation }\end{array}$} & \multicolumn{2}{c|}{ Fixation time } \\
\cline { 3 - 6 } & & $\mathrm{M}$ & $\mathrm{SD}$ & $\mathrm{M}$ & $\mathrm{SD}$ \\
\hline \multirow{3}{*}{ Manual } & top & 8.93 & 4.83 & 2.58 & 0.98 \\
\cline { 2 - 6 } & middle & 10.30 & 4.19 & 3.86 & 1.30 \\
\cline { 2 - 6 } & bottom & 6.05 & 3.10 & 1.04 & 0.79 \\
\hline \multirow{3}{*}{ Web page } & top & 9.58 & 5.23 & 2.07 & 0.95 \\
\cline { 2 - 6 } & middle & 10.53 & 6.41 & 3.07 & 1.53 \\
\cline { 2 - 6 } & bottom & 6.44 & 3.08 & 0.96 & 0.68 \\
\hline
\end{tabular}

The number of fixations was analyzed by variance analysis, we found: (1) The main effect of the format is not significant, $\mathrm{F}=1.037, \mathrm{p}>0.05$, that is, there was no difference in the number of fixations eyes on the brochure and web pages. (2) The main effect of propaganda content location is significant, $\mathrm{F}=3.556, \mathrm{p}<0.05$. Further examination found that there was no significant difference between the number of fixations on the top of the propaganda content and the number of fixations in the middle, a significant difference between the number of fixations eyes on the top and that of at the bottom, a significant difference between the number of fixations at the bottom and the number of fixations in the middle.

The fixation time was analyzed by variance analysis, we found: (1) The main effect of the format is not significant, $\mathrm{F}=0.123, \mathrm{p}>0.05$, that is, there was no difference in the fixation time between the manual and the web page. (2) The main effect of propaganda content location is significant, $\mathrm{F}=10.655, \mathrm{p}<0.05$.After the test, there was no significant difference between the upper fixation time and the middle fixation time, a significant difference between the upper fixation time and the fixation time at the bottom, a significant difference 
between the middle fixation time and the fixation time at the bottom.

\section{DISCUSSION}

\section{A. The Influence of Different Formats on the Memory of Content}

The statistical results of different formats are shown in the experiments that manual format is better than the webpage format for presenting propaganda content. Analyze the reasons: (1) the manual is usually composed of multiple pages, the content of each page is less, font size is different, the color of the text is not the same, but also with some pictures. A study of reading shows that when there is a correlation between the picture and the content of the article, there is a mutual promotion between them, and this can help subjects remember the content [7]. But the web pages are usually made by one page, the content of the text is much, the color of the text is unified, and it is easy to cause visual fatigue. Therefore, it is unfavorable to memory the content. (2) People prefer reading paper materials, this is mainly due to the series of electronic display features will affect people's cognitive activities, especially visual processing, such as the electronic screen refresh, brightness and contrast between the target and the background. And this can also cause greater cognitive and working memory load, making it easier for people to feel tired [8]. Although people are more and more contact with electronic information, but still more accustomed to reading paper materials.

\section{B. The Influence of Different Locations on the Memory of Content}

In daily reading, people usually start from the upper left corner, from left to right, from top to bottom. A study found that regardless of the text material and graphic material, if the presentation position is different, the reader's reading is different. It has been found that, regardless of the amount of material content, the reader's cognitive effect on the content of the material is affected by the position of presentation [9]. Usually, more attention are paid to the contents of the page top, the bottom of the page is rarely noticed.
In the experiment, the main task of the subject is to read the content of the manual and the page. The contents of the manual and web pages are processed under the condition of no attention, this design is in line with the habit of reading books and web pages in daily life. The results showed that no matter the number of fixation or fixation time, the position effect was significant. Specifically, the subjects were more interested in reading the contents of the top of the manual and the page and the content in the middle. The number of fixations on the top and in the middle is more than the number of fixations at the bottom, and the duration of fixation on the top and in the middle was longer than that of the bottom. The results show that the subjects had a deeper processing level on the top and middle of the page.

The recognition results show that the recognition effect of propaganda content on the top and middle of the page is the best, but that at the bottom of the page is the worst.

\section{V.CONCLUSION}

Under the experimental conditions, the following conclusions can be drawn: (1) the memory effect of the content that are presented by manual is significantly better than the web page. (2) The location of the propaganda content has certain influence on the eye movement pattern and the memory performance. In terms of eye movements, there are significant differences between the number of fixation and fixation time, that is, the number of fixation and fixation time on the top and middle of the page is significantly higher than that of lower part. Memory scores also showed significant differences between the central and upper, middle and upper memory effect is better.

Therefore, it is best for coal mine enterprise to use a safety culture manual to spread safety knowledge. Safety manual is easy to remember, and easy to carry, easy for employees to read. Moreover, the important publicity should be arranged on the middle-upper part of the manual at design time. In addition to the content of the text, related pictures should also be inserted, graphic 
combination, so as to achieve better publicity effect.

\section{REFERENCES}

[1] Kerr M A, Symons S E. Computerized presentation of text: Effects on children's reading of informational material [J]. Reading and Writing, 2006, 19(1):1-19.

[2] Noyes J M, Garland K J. VDT versus paper-based text: Reply to Mayes, Sims and Koonce [J]. International Journal of Industrial Ergonomics, 2003, 31 (6):411-423.

[3] Wang Aihua, Shu Hua. Effects of presenting mode of reading material on children reading activity [J]. Psychological Science, 2008, 31(2): 438-440.

[4] Noyes J M, Garland K J. Computer-vs. paper-based tasks: Are they equivalent?[J]. Ergonomics, 2008, 51(9): 1352-1375.
[5] Lohse G L, Wu D J. Eye movement patterns on Chinese yellow pages advertising [J]. Electronic Markets, 2001, 11(2): 87-96.

[6] Cheng li, Yang Zhiliang, Wang Xinfa. A study on eye movements of different presentations of web-ads [J]. Psychological Science, 2007, 30(3): 584-587.

[7] Shen Deli, Tao Yun. A study of eye movements in reading texts with or without illustrations for junior high school students [J]. Psychological Science, 2001, 24(4): 385-389.

[8] Wästlund E, Reinikka H, Norlander T, et al. Effects of VDT and paper presentation on consumption and production of information: Psychological and physiological factors[J]. Computers in Human Behavior, 2005, 21(2): 377-394.

[9] Du Han, Pang Bo, Chen Jiyue, et al. The study of comparing online advertising with newspaper advertising in different presentation conditions [J]. Psychological Exploration, 2013, 33(4): 380-38 